\title{
Rescheduling Railway Timetables in Presence of Passenger Transfers Between Lines Within a Transportation Network
}

\author{
Juan A. Mesa, Francisco A. Ortega, Miguel A. Pozo and Justo Puerto
}

\begin{abstract}
The problem of coordinating transfers consists of determining timetables which ensure the transfer of passengers between trains from different line runs at interchange stations. Two strategies can be considered: (1) Forcing the line runs to be synchronized; that is, a solution can be accepted only if there exists a connection between them, while the goal is minimizing travel times for passengers by using the minimum number of vehicles needed. (2) Minimizing an objective function that penalizes the lack of synchronization between line runs. The problem of transfer coordination turns out to be NP-hard even in the simple case of periodic timetables. Therefore, the problem is usually treated sequentially in two stages: first, determine the frequency of service according to the rate of demand, and then solve the problem of coordination by means of heuristics. This chapter considers a transit line where a train fleet circulates and stops at the stations according to a predetermined timetable which is known by the users. At any instant, passengers arrive at different stations in order to board these vehicles according to an assumed deterministic model of arrivals. In this scenario, a service rescheduling forced by an incidence is determined in order to minimize the loss of passengers who require transfers between different lines at the interchange stations. A case study consisting of a railway line with several equi-spaced stations, where it is possible a connection to
\end{abstract}

\section{J. A. Mesa}

Higher Technical School of Engineering, University of Seville, Camino de los Descubrimientos s/n, 41092 Seville, Spain

e-mail: jmesa@us.es

F. A. Ortega $(\bowtie)$

Higher Technical School of Architecture, University of Seville, Av. Reina Mercedes 2

41012 Seville, Spain

e-mail: riejos@us.es

M. A. Pozo - J. Puerto

Faculty of Mathematics, University of Seville, c/Tarfia s/n, 41092 Seville, Spain

e-mail: miguelpozo@us.es

J. Puerto

e-mail: puerto@us.es 
other lines at intermediate stations is analyzed for different scenarios where the loss of transfers is penalized.

Keywords Transit network - Transport scheduling • Disturbance management • Schedule synchronization

\section{Introduction}

Timetable design is a central problem in railway planning with many interfaces with other classical problems: line planning, vehicle scheduling, and delay management. The single-line Train Timetabling Problem (TTP) is devoted to obtaining and optimizing timetables of periodic and non-periodic heterogeneous trains that share a railway line with single and multiple track sections.

Given a railway infrastructure provided with different sections along a single transit line, the Train Timetabling Problem (TTP) consists of computing timetables that satisfy existing constraints and that optimize a single/multicriteria objective function for trains of both, passengers and cargo. The railway line may be occupied by other trains whose priority is higher than that of the new ones, and the new trains to be added may belong to different train operators. The requirement for periodicity of the timetables leads to the classification of TTP into Periodic (or cyclic) Train Timetabling and, on the other hand, Non-Periodic Train Timetabling.

In Periodic Timetabling, the timetable is easy to remember for the passengers although its solutions can become inefficient when planning resources such as crews and rolling stock. The mathematical model called Periodic Event Scheduling Problem (PESP) by Serafini and Ukovich [16] is the most widely used in the literature. In PESP, the events are scheduled for one cycle in such a way that the cycle can be repeated according to periodic time windows constraints. The PESP model has been used by authors in [10, 14, 15]. Interesting contributions on efficient railway operation management, oriented to European real contexts, can be found in the ARRIVAL project (http://arrival.cti.gr/, 2009).

Non-Periodic Train Timetabling is especially relevant on heavy-traffic, longdistance corridors where the capacity of the infrastructure is limited due to great traffic densities, as well as in presence of disturbances that can affect to the operativeness of train transit. The non-periodic train timetabling problem has been considered by most authors: [1, 2, 4, 5, 7-9, 11, 13, 17, 18].

Planners usually use running maps as graphic tools to help them in the planning process. A running map is a time-space diagram where possible crossings of trains can be observed. Figure 1 shows a time-space diagram that synthesizes the train expeditions of a piece of the $\mathrm{C} 4$ line that belongs to the Madrid commuter railway network (see Fig. 2). The names of the stations (cantons where passengers can board or alight on/from trains) are presented on the left side, and the vertical line represents train speeds when it passes through the sequence of tracks between consecutive stations. 


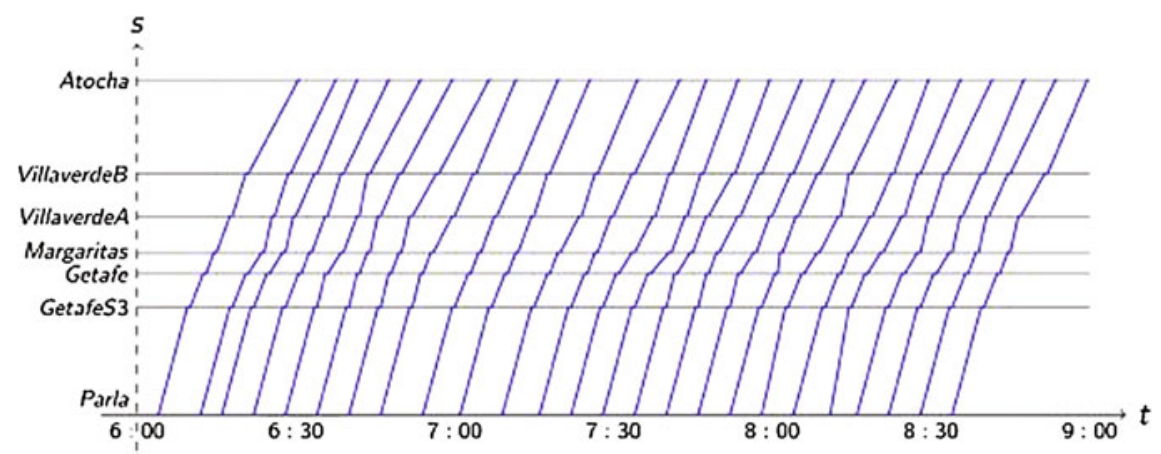

Fig. 1 Twenty-five instances of train schedules along the transit corridor

Fig. 2 Line C4 (ParlaAtocha)

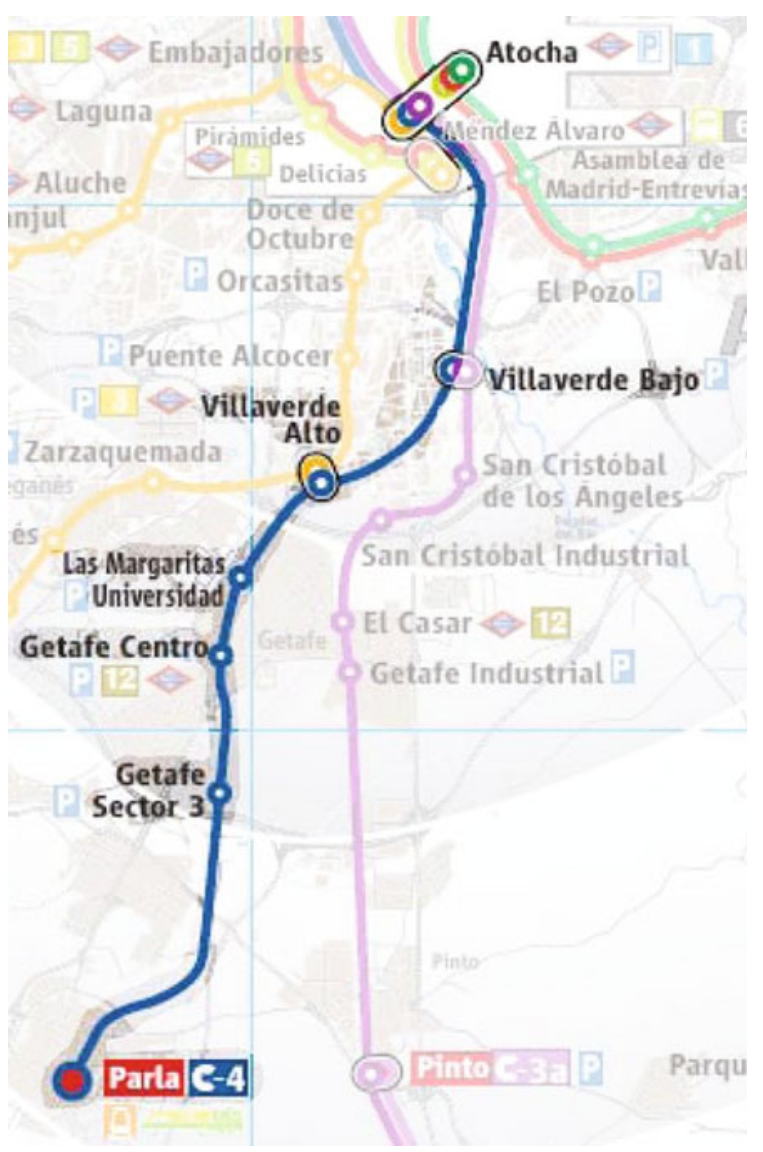

The slope of the polygonal line associated with a train corresponds with the commercial speed of that train (with a stop between stations), and the horizontal segments can be viewed as the stopping time at stations. A transit corridor of high 
traffic density will generate in a labyrinthine tangle of polygonal lines, each of which will correspond to the hours of operation of a train, making infeasible a nonautomated assessment of the possible alternatives.

A conflict of crossing between trains could take place along the transit corridor. Graphically, a conflict can be represented by means of a pair of two rectilinear segments that intersect in the same canton. If such canton had multiple pathways, it is possible to solve the conflict by imposing a waiting time to some of trains.

In order to be feasible, a timetable has to fulfill a set of constraints that can be classified into three main groups, depending on whether they are concerned with:

- User Requirements (parameters of trains to be scheduled): time windows for departure and arrival times, maximum delay.

- Traffic constraints: running time, crossing, commercial stop, overtaking on the track section, delay for unexpected stop, reception time, expedition time, simultaneous departure.

- Infrastructure constraints: network topology, finite capacity of stations, closing time, headway time.

Many references consider Mixed Integer Problem formulations in which the arrival and departures times are represented by continuous variables and there are binary variables expressing the order of the train departures from each station. The variables chosen to formulate the model must be able to formally express these restrictions, so that only feasible timetables can be considered as possible solutions. There are two main criteria to assess the quality of the solutions: Minimize operating costs (point of view of the operator) and Minimize riding and transfer times (perspective of passengers). Moreover, other complementary objectives can be used, for example: minimize the passenger waiting time in the case of changeovers, balance the delay of trains in both directions, minimize the average delay of new trains with respect to their optimum, etc. Accidents, strike days and other sources of train delays or cancellations force to modify the scheduled timetable when trains in some sections cannot run according to the initial planning. Rescheduling is the process of updating an existing production plan in response to disruptions or other changes [19]. Rescheduling timetables is especially important in heavily used areas because individual events (delays) can easily impact many other trains causing secondary delays to ripple through the network. In order to manage this domino effect when a train is late and reduce the impact on the other trains, controllers must manually adjust the routing of trains. The effectiveness of the rescheduling and train control system at reducing total delay is highly dependent on the specific circumstances (timetable, train routes, topology of the station and occupation of those tracks located before in the bottleneck area). The modification to the timetable should be performed without introducing inconsistencies. In that case, assessing the feasibility of any modification of the existing timetables will necessarily require a computer-aided procedure. In terms of railway operation production plans, the main decisions that must be addressed in the rescheduling process are: 
- Provide new reference times for all trains located at specific points in the network (downtime of trains at stations and reference speed on open track).

- Re-routing trains.

- Re-allocate available resources (staff, rolling stock).

The results presented in this chapter are focused mainly on retiming trains that remain operative (the above first point). For that purpose, it will be necessary to change the departure or arrival times at stations and other reference points. Decisions can also include rerouting trains in affected areas by means of cancelling trains, adding supplementary stops or short turns.

Research on rescheduling algorithms has been underway for many years. See, for example, the survey paper by Cordeau et al. [6] and the recent contribution of Canca et al. [3]. In order to successfully use rescheduling algorithms in dense railway networks, it is necessary to analyze the whole production process to determine how new schedules can be most efficiently implemented. The time it takes to complete a rescheduling process (from the point of time when a given threshold is exceeded until the new production plan is applied) for a large network leads to three important questions regarding implementation of the process. Namely, rescheduling: should it be periodic; could it be interrupt-able; should it be implemented in spite of being an infeasible plan?

Each of these questions must be clarified before to completing the rescheduling process. In this chapter, we present an approach to generate acyclic timetables for single line track. This approach is based on geometrical properties associated to topology of a transit corridor.

The chapter is organized as follows. In Sect. 2, a model of graphical representation for train schedules is introduced and a procedure for estimating the number of users associated to feasible schedules is developed. The formulation of the decision model is introduced in Sect. 3, in addition to the subsequent extended scenarios, where transfers between lines that cross the transit corridor are taken into account. Finally, some conclusions are summarized in Sect. 4.

\section{Discretizing Time Horizon and Weighting the Feasible Schedules}

\subsection{A Graphical Schedule Representation Based on Timetable-Points}

According to Mesa et al. [12], we assume a canonical time unit $h>0$ (time taken to travel without stopping between two consecutive sections) for generating a uniform mesh of squares of length $h$ in the first quadrant, that can be used to represent the activity map of trains at section (or station) $k$. Inside this activity map of the $k$-th stretch/station, each point will indicate the arriving time ( $x$-coordinate) and the leaving time ( $y$-coordinate) of a train. Arrival time of trains can be seen in 
Fig. 3 Three trains passing through the station $k$

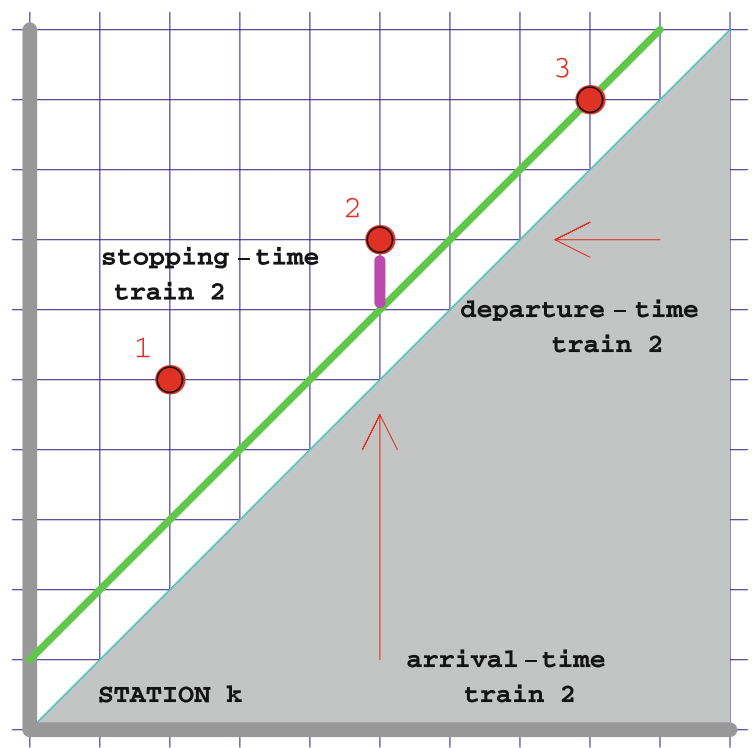

the horizontal axis, while the projection on the vertical axis represents its departure time. In a generic $k$-station, each train has assigned a unique point (timetable-point, in the following) whose coordinates must be necessarily located in the upper triangle of the first quadrant above the straight line $y=x+h$ (the upper subdiagonal outlined in green). For instance, Fig. 3 shows data corresponding to three different trains. The first one spends a time equivalent to $2 h$ in boarding and alighting passengers. Train 2 uses the minimum time required for that operation, i.e. $h$. Finally, train 3 does not stop at station $k$, hence its position is located on the displaced diagonal in the first quadrant.

The sequence of sections (with stops or not for passengers) along the railway line will correspond to a succession of temporary diagrams, where active timetable-points will indicate real arrival-departure timetables. Each timetable-point in the $k$-th diagram of activity will match to some other feasible timetable-point of the vertical segment that starts from its projection on the diagonal in the $(k+1)$-th activity-map. Moreover, since activity maps have the same homogeneous structure for all stations, a line run can be graphically viewed as a non-decreasing polygonal line that crosses through the sequence of temporary diagrams corresponding to the corridor stations, visiting an only timetable-point per diagram.

\subsection{Assuming a Pattern for the Demand Behavior}

Assume that arrival/departure times of trains at stations were previously set and are known by users. In that case, we can ensure that these temporal marks mobilize a population of potential travelers towards the station platform, converging in time 
Fig. 4 Usual demand behavior in terms of percentage of user's presence at platform
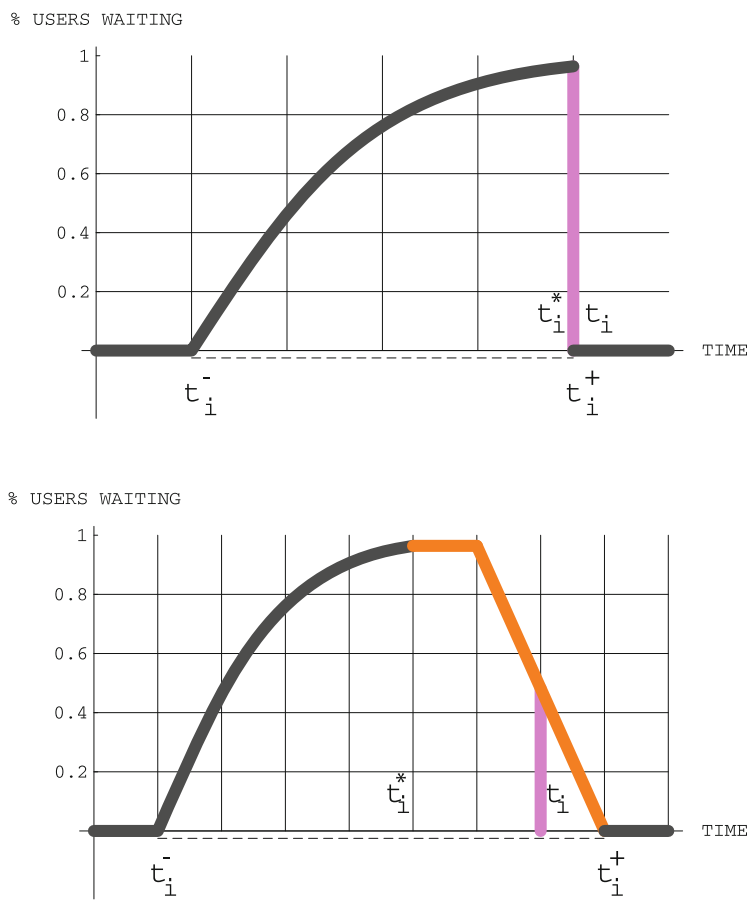

Fig. 5 Demand behavior when train is delayed

with the timely arrival of such train. Figure 4 explains in percentage terms the traveler accumulation on the platform of the station $k$, due to the imminent arrival of the scheduled train at time $t_{i}$. Time interval associated with the arrival of travelers and their consequent accumulation in the platform is denoted by $\left[t_{i}^{-}, t_{i}^{+}\right]$.

Before arriving train $t_{i}$ at station $k$, the number of users that reaches the platform with the purpose of boarding on the train is increasing until shortly before the estimated time of train arrival. If the train arrived on time, the whole population placed on platform could be transported as shows Fig. 4. Nevertheless, if train $t_{i}$ were to be delayed, the reaction of users when they know the existence of such delay would consist of initially waiting along a short certain period of time. Subsequently, the curve that models the percentage of population waiting would appear stabilized. After this period, the traveler population gradually decreases until disappearing. If the train arrived late, only a portion of the population that normally waits could be transported (Fig. 5).

Finally, if the train arrived and departed in advance, only users who were already placed on the platform could take the train. The other passengers will be coming in the usual way, because they were unaware of this schedule change (Fig. 6). The option to wait a certain period of time leads to the possibility of taking the next train.

As illustration, a study case composed of a railway line with several equispaced stations, separated from each other by a distance (travel time) equal to $2 \mathrm{~min}$, is considered. It has been assumed an operational time corresponding to the 
Fig. 6 Demand behavior if train departed in advance

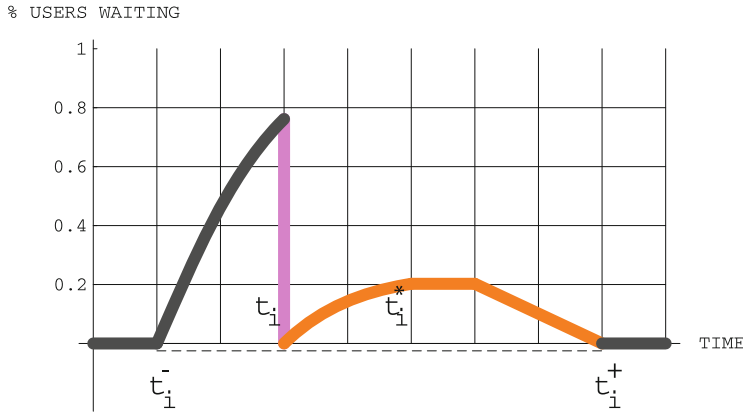

Table 1 Arrival/departure times associated to trains at stations

\begin{tabular}{llllllll}
\hline Station & 1 & 2 & 3 & 4 & 5 & 6 & 7 \\
\hline Train 1 & $8: 26 / 8: 28$ & $8: 30 / 8: 32$ & $8: 34 / 8: 36$ & $8: 38 / 8: 42$ & $8: 44 / 8: 48$ & $8: 50 / 8: 52$ & $8: 54 / 8: 56$ \\
Train 2 & $8: 38 / 8: 40$ & $8: 42 / 8: 44$ & $8: 46 / 8: 48$ & $8: 50 / 8: 54$ & $8: 56 / 9: 00$ & $9: 02 / 9: 04$ & $9: 06 / 9: 08$ \\
Train 3 & $8: 50 / 8: 52$ & $8: 54 / 8: 56$ & $8: 58 / 9: 00$ & $9: 02 / 9: 06$ & $9: 08 / 9: 12$ & $9: 14 / 9: 16$ & $9: 18 / 9: 20$ \\
\hline
\end{tabular}

Table 2 Passengers boarding to train at stations

\begin{tabular}{llllllll}
\hline Station number & 1 & 2 & 3 & 4 & 5 & 6 & 7 \\
\hline Train 1: Passengers & 1,417 & 1153 & 664 & 281 & 77 & 39 & 0 \\
Train 2: Passengers & 1,143 & 756 & 359 & 113 & 23 & 10 & 0 \\
Train 3: Passengers & 2,131 & 1,204 & 488 & 117 & 18 & 7 & 0 \\
\hline
\end{tabular}

morning interval (8:20-9:30) with partitions of size $h$ (1 min). Initially, there are three vehicles to run along the line and the arrival/departure time at stations are known by users (Table 1).

In the trip distribution along the corridor, it has been assumed that the first stations are mainly trip generators, while the ending station is an attractive destination. In real instances, this setting is commonly associated to a transit line which connects far residential areas with the city center. Attractiveness levels between the first nodes and the final station have been assumed to be decreasing with respect to the distance between them. According to the above consideration, a time-dependent origin-destination matrix has been randomly built for a population of 10,000 users. From it, the number of users accessing each train station is shown in Table 2.

Assume that, as consequence of an incident, the system operator must reduce the fleet size by one unit. Rescheduling train timetables must minimize the loss of users, by introducing advances or delays in the original schedules of the two vehicles which will remain operative. According to the model proposed in the article, the following distribution of passengers that access to stations is shown in Fig. 7 and, if train were not punctual, population waiting for boarding can be deterministically estimated (Fig. 8). Since it is assumed that the user loss for 


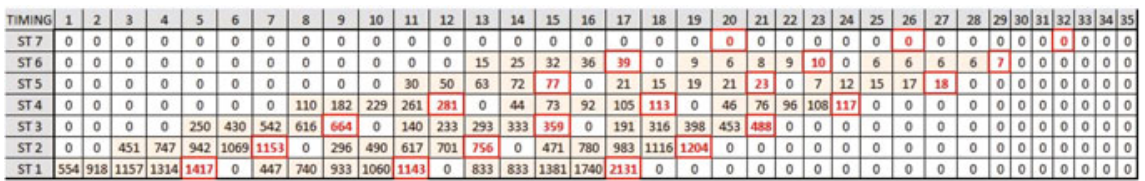

Fig. 7 User's presence at platform waiting a punctual arrival

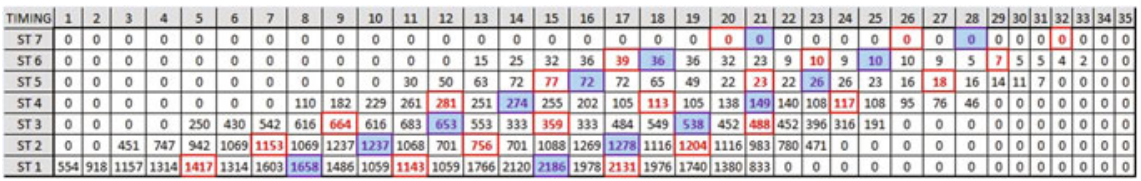

Fig. 8 User's presence at platform waiting a delayed arrival

railway system is only caused by decisions of putting advanced or delayed schedules, the sequence of blue cells indicates optimal reprogramming of the two feasible schedules.

If the solution obtained by applying this methodology $(8,127$ user served) is compared with the result obtained when the train that serves to the least number of users is cancelled $(7,596)$, passenger loss is reduced by six.

\section{Model Formulation}

Assuming the previous pattern, a new timing for train arrivals at stations along the transit line can be determined in order to take advantage of overlapping demand curves generated from neighboring timetable-points. As a result of a disruption on the network, the subsequent rescheduling of train timetables can be based in this fact with the aim of minimizing the loss of passengers. Two scenarios can be considered, depending on that passengers can (or not) require transfers toward/ from other network lines at particular times.

\subsection{Scenario 1: Without Transfers}

\subsubsection{Indices and Sets}

$i \in I \quad$ index identifying trains of set $I$ which run along the transit corridor

$k \in K \quad$ index identifying cantons (or stations) of set $K$

$u, v \in T \quad$ indices identifying the discrete time horizon $T$

$(u, v) \in M_{k} \quad$ coordinates corresponding to temporary map $M$ at station $k$. 


\subsubsection{Parameters}

$a_{v}^{i k} \quad$ user population available to boarding train $i$ at station $k$ and at time $v$.

\subsubsection{Decision Variables}

$x_{u v}^{i k} \quad$ binary variable equals to 1 if train $i$ is located at point $(u, v)$ at station $k 0$, otherwise.

\subsubsection{Formulation of the Model Without Transfers}

$$
\max \sum_{i \in I} \sum_{k \in K} \sum_{(u, v) \in M_{k}} a_{v}^{i k} x_{u v}^{i k}
$$

s.t.

$$
\begin{gathered}
\sum_{i \in I} \sum_{(u, v) \in M_{k}} x_{u v}^{i k}=|I| ; \quad k \in K \\
\sum_{k \in K} \sum_{(u, v) \in M_{k}} x_{u v}^{i k}=|K| ; \quad i \in I \\
\sum_{i \in I} \sum_{u^{\prime}<v} x_{u^{\prime} v}^{i k} \leq 1, \quad \sum_{i \in I} \sum_{v^{\prime}>u} x_{u v^{\prime}}^{i k} \leq 1 ; \quad(u, v) \in M_{k}, \quad k \in K \\
x_{u v}^{i k} \leq \sum_{v^{\prime}>v} x_{(v+1) v^{\prime}}^{i(k+1)} ; \quad(u, v) \in M_{k}, \quad k \in K(k \neq|K|) \\
\sum_{i \in I} \sum_{u^{\prime}<v, v^{\prime}>u} x_{u^{\prime} v^{\prime}}^{i k} \leq n_{k}-x_{u v}^{i k} ; \quad(u, v) \in M_{k}, \quad k \in K \\
x_{u v}^{i k} \in\{0,1\} ; \quad(u, v) \in T, \quad i \in I, \quad k \in K
\end{gathered}
$$

The objective function maximizes the number of users that can be transported along the rail corridor, picking them up at their respective stations during the time interval that they are waiting on platforms. In this sense, the objective function (1) maximizes the mobility of customers that use the railway system. Constraints (2) establish that the number of train schedules to be located must be exactly $|I|$. Restrictions (3) force passage through each station (with or without stopping) for all trains to be determined. Constraints (4) indicate that there can be no train arriving/departing from the $k$-th station if there was just another train operating. Restrictions (5) establish that if there is a timetable-point located at position 
$(u, v)$ of the temporary map for the $k$-th station, then there must be another timetable point, at the $(k+1)$-th station, on the $v$-th column. Limitation of the number of trains that can operate, according to the existing number of tracks, is indicated by means of constraints (6). Finally, restrictions (7) state the binary nature of the decision variables of integer linear programming model.

From the temporary map corresponding to the first station to that of the last station (respectively labeled 0 and $|K|$ ), a directed acyclic graph $G=(V, E)$ can be built connecting feasible sequence of timetable-points corresponding to adjacent activity maps. Nodes $Q_{k}\left(u_{k}, v_{k}\right)$ and $Q_{k+1}\left(u_{k+1}, v_{k+1}\right)$ are connected by an arc of set $E$ if both timetable-points satisfy the model constraints (2)-(6). By means of this geometric meaning, the determination of an optimal train timetable will be equivalent to find an optimal solution to the problem of locating a sequence of $|K|$ timetable-points (one for each temporary map) which maximizes the user mobility. Subsequently, the application of a longest path algorithm on graph $G=(V, E)$ will generate an effective schedule for the line. This decision model uses $|J| \cdot|K| \cdot|M|$ variables, where $|M|$ represents the maximum number of feasible timetable-points $(u, v)$ in the upper triangles of temporary maps associated to stations of corridor.

\subsection{Scenarios Preserving/Rewarding Transfers Between Transit Lines}

If transit corridor intersects with others transit lines at specific stations, as is shown in Fig. 9, the determination of new timetables should ensure the transfer of passengers between trains from different line runs at such interchange stations.

Two strategies can be considered:

- Imposing synchronization between the timetables of these lines; that is, a solution can be accepted only if the connection between them is feasible (Scenario 2.1).

- Rewarding the possibility of providing transfers for passengers of external lines towards concurrent expeditions of the internal line by means of a weighting factor $\gamma>1$ (Scenario 2.2).

The geometrical model above developed can be slightly adapted in order to catch the consideration of transfers. For this purpose, we define different new indices and parameters. Let $j \in J$ be the index that identifies trains of other transit lines concurrent with lines runs of set $I$. Let $s \in S \subset K$ be the index that enumerates the subset of stations that allow transfers to the travelers. Let $F_{s}(u, v) \subset M_{s}$ be the subset of timetable-points in the temporary map $M$ of station $\mathrm{s}$ where transfers between two transit lines can be carried out. For instance, Fig. 10 shows the timetable-point (filled in red) of another line (line A) when arrives/ departs at/from station 4 at times $u=4$ and $v=8$, respectively. If the synchronization between the timetables of these lines were imposed, the feasible subset of 
Fig. 9 Corridor intersected with external lines

Fig. 10 Candidate locations for timetable-points which ensure transfers
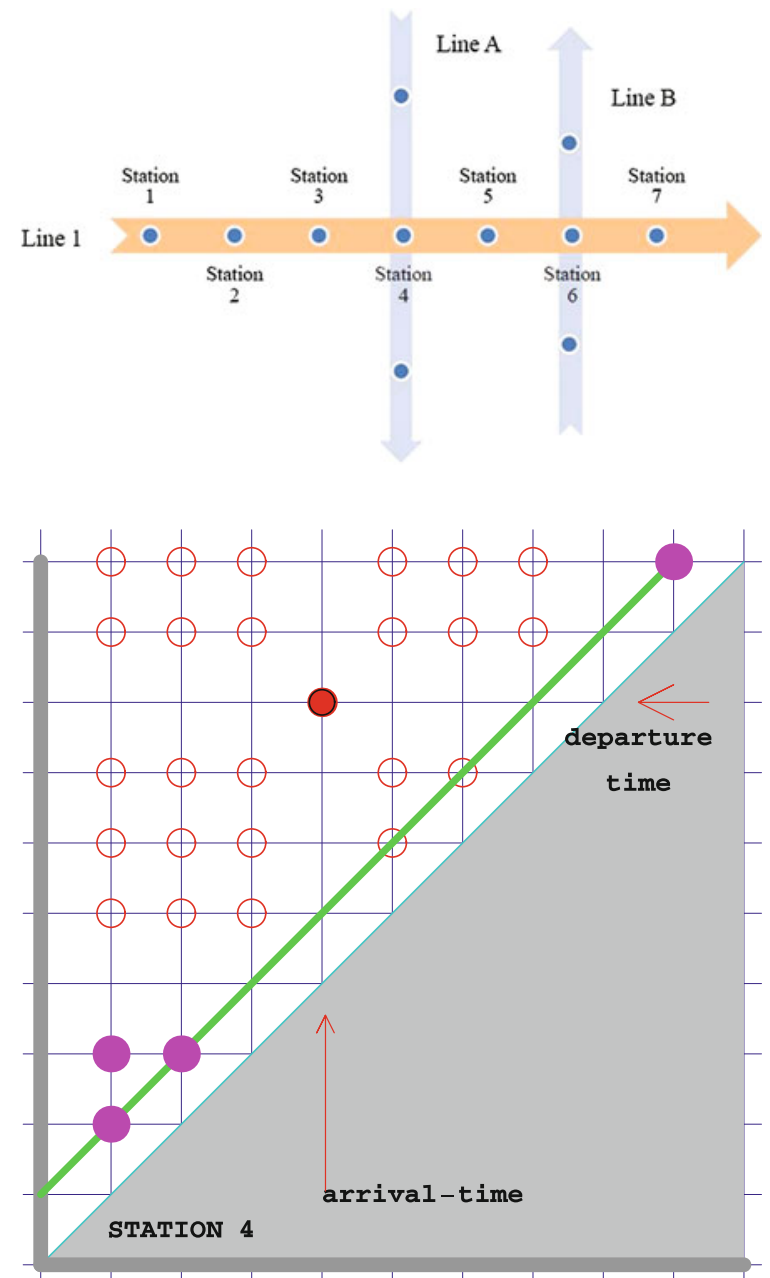

timetable-points (i.e., $F_{s}(4,8)$ ), where transfer is preserved, would coincide with the set of unfilled points in magenta color. Consistently with the notation used for decision variables in the model, let $y_{u v}^{j s}$ be a binary input data which is equal to 1 if train $j$ (of an external line whose arrival/depart timetables are given) is located at timetable-point $(u, v)$ at station; otherwise, its value would be 0 .

Scenario 2.1: Constraints (8) establish that if there is an active (i.e., $y_{u v}^{j s}=1$ ) timetable-point located at position $(u, v)$ of the temporary map for the $s$-th station of an outside line, then there must be at least another active timetable-point at the same station for synchronizing transfers from/toward line runs $i$ of the inner transit line $I$.

$$
y_{u v}^{j s} \leq \sum_{i \in I} \sum_{\left(u^{\prime}, v^{\prime}\right) \in F_{s}(u, v)} x_{u^{\prime} v^{\prime}}^{i s} ; \quad j \in J,(u, v) \in M_{s}, s \in S
$$


Therefore, objective (1) and constraints (2)-(8) constitute a procedure for maximizing the number of passengers who enter in the system after rescheduling, once transfers towards other external lines is preserved.

Scenario 2.2: For this context, it is necessary to distinguish between users who enter in the system from outside and passengers who previously entered into the system with the certainty of being able to make a transfer to another line already. Objective to maximize must take into account this division of populations and asymmetrically favor one over the other population by using a weighting factor $\gamma>1$. Let $b_{u v}^{j k}$ be a real input data which represents the population available to transferring from train $j$ at station $k$ and at timetable-point $(u, v)$. The objective (1) after being modifying is

$$
\max \sum_{i \in I} \sum_{k \in K} \sum_{(u, v) \in M_{k}}\left(a_{v}^{i k}+\mu \sum_{j \in J} \sum_{\left(u^{\prime}, v^{\prime}\right) \in F_{k}(u, v)} b_{u^{\prime} v^{\prime}}^{j k}\right) x_{u v}^{i k}
$$

We must remark that if $k$ is not an interchange station, then $F_{k}(u, v) \equiv \varnothing$ and the second additive term is cancelled. Therefore, objective (1) and constraints (2)-(8) constitute a procedure for maximizing mobility of travelers who enter in the system after rescheduling, by ensuring the option of transferring from/towards other external lines at interchange stations.

\section{Conclusions}

A geometric approach to determine the redistribution of service along a rail corridor has been introduced. Motivation for rescheduling railway timetables is caused by the forced reduction of fleet size due to accidents, strikes and other sources of train delays and cancellations. Two scenarios have been presented: a context without considering transfers from/towards other transit lines, and a setting where the existence of transfers between lines must be preserved although the service would have been rescheduled. In the second case, two scenarios have been formulated taking or not into consideration an equitable evaluation between the different collective of users. A common approach for these scenarios has been developed by using a geometrical representation of train timetables at stations. The associated formulations are Integer Linear Programming models, where the number of decision variables can be reduced according to different constraints imposed by the structural and fleet capacities. The theoretical development has been illustrated with a non-sophisticated example in order to clarify the concepts used through the chapter.

Acknowledgments This work was partially supported by Ministerio de Economía y Competitividad (Spain)/FEDER under grants MTM2010- 19576-C02-01 and MTM2012-37048, and by Junta de Andalucía (Spain)/FEDER under excellence projects P09-TEP-5022, FQM-5849 and P10-FQM-5849. 


\section{References}

1. Barber, F., Ingolotti, L., Lova, A., Tormos, P., Salido, M.A.: Meta-heuristic and constraintbased approaches for single-line railway timetabling. Lect. Notes Comput. Sci. 5868, 145-181 (2009)

2. Cai, X., Goh, C.J.: A fast heuristic for the train scheduling problem. Comput. Oper. Res. 21, 499-510 (1994)

3. Canca, D., Barrena, E., Zarzo, A., Ortega, F.A., Algaba, E.: Optimal train reallocation strategies under service disruptions. Proc. Soc. Behav. Sci. 54, 402-413 (2012)

4. Caprara, A., Monaci, M., Toth, P., Guida, P.: A lagrangian heuristic algorithm for a realworld train timetabling problem. Discr. Appl. Math. 154, 738-753 (2006)

5. Carey, M., Lockwood, D.: A model, algorithms and strategy for train pathing. J. Oper. Res. Soc. 46, 988-1005 (1995)

6. Cordeau, J., Toth, P., Vigo, D.: A survey of optimization models for train routing and scheduling. Trans. Sci. 32, 380-404 (1998)

7. Higgins, A., Kozan, E., Ferreira, L.: Heuristic techniques for single line train scheduling. J. Heuristics 3, 43-62 (1997)

8. Ingolotti, L., Lova, A., Barber, F., Tormos, P., Salido, M.A., Abril, M.: New heuristics to solve the CSOP railway timetabling problem. Lect. Notes Comput. Sci. 4031, 400-409 (2006)

9. Jovanovic, D., Harker, P.T.: Tactical scheduling of rail operations: the SCAN-I system. Trans. Sci. 25, 46-64 (1991)

10. Kroon, L., Peeters, L.: A variable time model for cycling railway timetabling. Trans. Sci. 37, 198-212 (2003)

11. Mesa, J.A., Ortega, F.A., Pozo, M.A.: Effective allocation of fleet frequencies by reducing intermediate stops and short turning in transit systems. Lect. Notes Comput. Sci. 5868, 293-309 (2009)

12. Mesa, J.A., Ortega, F.A., Pozo, M.A.: A geometric model for an effective rescheduling after reducing service in public transportation systems. Comput. Oper. Res. 40, 737-746 (2013)

13. Michaelis, M., Schöbel, A.: Integrating line planning, timetabling, and vehicle scheduling: a customer-oriented heuristic. J. Public Transp. 1, 211-232 (2009)

14. Nachtigall, K., Voget, S.: A genetic algorithm approach to periodic railway synchronization. Comput. Oper. Res. 23, 453-463 (1996)

15. Odijk, M.: A constraint generation algorithm for the construction of periodic railway timetables. Trans. Res. B 30, 455-464 (1996)

16. Serafini, P., Ukovich, W.: A mathematical for periodic scheduling problems. SIAM J. Discr. Math. 2, 550-581 (1989)

17. Silva de Oliveira, E.: Solving single-track railway scheduling problem using constraint programming. Ph.D thesis, The University of Leeds, School of Computing (2001)

18. Szpigel, B.: Optimal train scheduling on a single track railway. In: Roos, M. (ed.) Proceedings of IFORS Conference on Operational Research 1972, pp. 343-352 (1973)

19. Vieira, G.E., Herrmann, J.W., Lin, E.: Rescheduling manufacturing systems: a framework of strategies, policies, and methods. J. Sched. 6, 39-62 (2003) 